\title{
Demographic and clinical phenotypic differences between people with dissociative seizures and those with other psychiatric disorders
}

\author{
Abigail Smakowski, Jacob S. Bird, Megan Pritchard and Marco Mula
}

\section{Background}

A large amount of literature surrounds the differences between dissociative neurological symptom disorder with non-epileptic seizures (DNSD-S) and epilepsy.

\section{Aims}

To explore the research gap on phenotypic differences between DNSD-S and other psychiatric disorders.

\section{Method}

We conducted a case-control study of 1860 patients $(620$ patients with DNSD-S and 1240 controls with other psychiatric disorders) seen at the South London and Maudsley Hospital NHS Trust between 2007 and 2019.

\section{Results}

Compared with the controls, the patients with DNSD-S were more likely to be female ( $76 \mathrm{v} .47 \%, P<0.001)$, of White ethnicity (77 v. 60\%, $P<0.001)$, married ( 34 V. 14\%, $P<0.001)$ and living in areas of lower socioeconomic status $(-3.79,95 \% \mathrm{Cl}-2.62$ to $-4.96, P<0.001)$. Two peaks for age at diagnosis were observed for DNSD-S: the early 20s and late 40s. After 31 years of age, men's chance of being diagnosed with DNSD-S increased from 19 to $28 \%(P=0.009)$. People with DNSD-S presented more commonly with a history of a neurological episodic or paroxysmal disorder ( $\mathrm{OR}=12,95 \% \mathrm{Cl} 7.82-20.26)$, another dissociative disorder $(\mathrm{OR}=10,95 \% \mathrm{Cl} 1.64-65.95)$ or unclassified signs or symptoms (OR $=4$, 95\% $\mathrm{Cl}$ 2.61-6.43). Neither anxiety, depression nor other somatoform disorders predicted subsequent diagnosis of DNSD-S, and controls had a larger proportion of preceding psychiatric diagnoses than patients with DNSD-S $(65 \mathrm{~V}$. $49 \%, P<0.001)$

\section{Conclusions}

This is the first study comparing demographic and phenotypic correlates of patients with DNSD-S against a large cohort of psychiatric patients. These data will inform development and drive service needs in psychiatry for people with DNSD-S.

\section{Keywords}

Non-epileptic seizures; dissociative disorders; neuropsychiatry; neuropsychology; psychopathology.

\section{Copyright and usage}

(c) The Author(s), 2021. Published by Cambridge University Press on behalf of the Royal College of Psychiatrists. This is an Open Access article, distributed under the terms of the Creative Commons Attribution-NonCommercial-NoDerivatives licence (http://creativecommons.org/licenses/by-nc-nd/4.0/), which permits non-commercial re-use, distribution, and reproduction in any medium, provided the original work is unaltered and is properly cited. The written permission of Cambridge University Press must be obtained for commercial re-use or in order to create a derivative work
Dissociative neurological symptom disorder with non-epileptic seizures (DNSD-S) is characterised by seizures or convulsions that are not consistent with a recognised disease of the nervous system, other mental or behavioural disorders or other health condition. Classified within the functional neurological disorders in DSM-5 and dissociative neurological symptom disorder in ICD-11, they are associated with a range of motor, sensory and mental manifestations and impaired self-control. Dissociative disorders have been reported as highly prevalent in psychiatric out-patient samples, and epidemiological studies have shown incidence rates for DNSD-S ranging between 1.5 and 4.9 per 100000 per year, with prevalence rates ranging between 2 and 33 per $100000 .{ }^{1,2}$ With one in five referrals to a first seizure neurology clinic due to DNSD-S, ${ }^{3}$ ultimately both neurologists and psychiatrists will find themselves responsible for explaining the diagnosis to patients. They will need to be equipped with knowledge to explain a referral to psychiatry and how DNSD-S is different from other more wellknown conditions in psychiatry, while providing an explanation of the mechanism behind how patients can develop physical symptoms through inorganic means. Clinicians will find it useful to give further reasons using an evidenced-based archetypal presentation, reassuring patients that they are not alone.

Despite increased interest into the aetiology of DNSD-S over the past decade, the bulk of the literature thus far has only focused on factors differentiating DNSD-S from epilepsy., ${ }^{4,5}$ This is valuable at a diagnostic level, as people with DNSD-S are often misdiagnosed with epilepsy and vice versa. However, DNSD-S is a psychiatric condition and the mainstay of treatment is psychiatric and psychological. ${ }^{6}$ For all these reasons, it is astonishing the limited amount of data about phenotypic differences between DNSD-S and other mental disorders. These data are of great relevance for care provision, planning and outcome studies. In addition, the majority of available studies are affected by a number of methodological limitations, including small samples and retrospective design, ${ }^{6,7}$ with only one study so far conducted at a population level. ${ }^{8}$ Goldstein and colleagues have taken the first step to characterising the typical presentation of people with DNSD-S. ${ }^{8}$ However, more data are needed to validate the preceding and predictive factors of DNSD-S in comparison with other psychiatric conditions. Findings suggest that comorbid neurological diseases occur in $10 \%$ of people with functional neurological disorders ${ }^{2}$ and comorbid epilepsy in $10-30 \%$ of people with DNSD-S. ${ }^{9}$ Research suggests that affective disorders can appear in $42 \%{ }^{10}$ and functional somatic symptoms in $66-$ $70 \%{ }^{11,12}$ of people with DNSD-S, with personality disorders also being common. ${ }^{13}$ Data on medical and psychiatric history research are available from several studies, yet all previous studies were conducted with small samples, or lacked a psychiatric control group or used people with epilepsy. In addition to this, most studies used a top-down design to probe for diseases, with researchers using selfreport or relying on experience to ask for the presence of specific conditions. It would be more robust to collect diagnostic data directly from clinical notes. This is a case-control study investigating the clinical characteristics of individuals with DNSD-S at diagnosis, in a large cohort of people with other mental disorders. 


\section{Method}

\section{Design, setting and data source}

Medical records of patients seen at the South London and Maudsley NHS Foundation Trust (SLaM) between 1 January 2007 and 6 November 2019 were analysed. SLaM is a National Health Service mental health trust that provides mental healthcare to a population of roughly 1.3 million residents in London, UK, as well as a national referral service. In 2007, the National Institute for Health Research (NIHR) Biomedical Research Centre at SLaM and King's College London developed the Clinical Record Interactive Search (CRIS) system. CRIS provides researchers with regulated access to anonymised information contained in SLaM's medical records, including over 250000 patients. ${ }^{14,15}$

CRIS was approved as a secondary data resource by the National Research Ethics Committee South Central Oxford C (ref: 18/SC/ 0372). The CRIS Research Oversight Committee granted our project access in March 2018 (project number 18-033).

\section{Participant selection}

Patients with DNSD-S were identified using CRIS by searching for the ICD-10 code F44.5 (Dissociative seizures) as a diagnosis. Patients for whom the code F44.5 was not the primary psychiatric diagnosis were excluded because a secondary diagnosis is not considered the main condition in a phenotype, nor as driving the major effect on the patient's life. Furthermore, we wanted to focus on DNSD-S as the primary problem from a psychopathological point of view, rather than conditions where DNSD-S could be considered the consequence of other major psychiatric conditions, thus masking the specific contribution of DNSD-S compared with a general sample of patients with psychiatric disorders. Inclusion of DNSD-S as a secondary diagnosis would have potentially biased the sample, especially because it is compared with a general sample of patients with psychiatric diagnoses. Cases and controls with missing demographic data were excluded. All patients with DNSD-S referred to SLaM already have a documented diagnosis of DNSD-S according to International League Against Epilepsy (ILAE) criteria ${ }^{11}$ made in an epilepsy centre.

For every DNSD-S 'case' we selected the two closest patients in time who were diagnosed in SLaM with a different primary psychiatric diagnosis (F code in ICD-10) on the day the 'case' received theirs. Controls were excluded if they had any history of 'dissociative seizures' and each control could only be used as a match once.

\section{Outcome variables}

We gathered demographic data regarding participant's gender, age, ethnicity, marital status, socioeconomic score and age at diagnosis. These variables were generally taken straight from CRIS. For temporal variables such as marital status, the most recent information at or before diagnosis was taken. For socioeconomic status, the active address on the 6 November 2019 or the most recent preceding address was used. Addresses were allocated to area scores according to the English Index of Multiple Deprivation $(2015)^{16}$, giving a score of 1 (most deprived) to 32844 (least deprived). Data on specific past psychiatric conditions were pulled from the CRIS database because we assumed that diagnoses delivered in a mental health service would be more accurate than diagnoses made in primary care. Data on past medical diagnoses were gathered by incorporating data from the Hospital Episode Statistics (HES) database (National Health Service, 2017). The HES database is linked with the CRIS database via a third-party clinical linkage data service using deterministic or probabilistic matching if/as required. ${ }^{15}$ This database contains details of all diagnoses, admissions and out- patient appointments at NHS hospitals across England. Owing to the vast number of conditions within medicine, we initially grouped medical diagnoses using the main chapters of ICD-10 to highlight any significantly associated medical categories. Given that past research has highlighted epilepsy in patients with dissociative seizures, we also broke down the G (neurology) ICD category to assess the predictivity of all neurological conditions.

\section{Statistical analysis}

All variables were analysed using IMB SPSS Statistics software, version 25 for Windows. Chi-square tests were used to compare gender, ethnicity, marital status and all medical and psychiatric history. $T$-tests were used to compare means of age at diagnosis and deprivation score. Exploratory analyses included chi-square comparisons within the DNSD-S group and between-group comparisons of age at diagnosis, split by the median. Binary logistic regression was used to further assess all significant variables from previous analyses to produce odds ratios.

\section{Results}

A total of 930 patients were identified to have a diagnosis of DNSD-S. Of these, 104 had DNSD-S as a secondary diagnosis and were therefore removed, leaving 826 in the final DNSD-S group. Statistical comparisons between the secondary diagnosis group and the final DNSD-S group showed no significant differences on all demographic variables apart from gender, where those with primary DNSD-S showed a greater proportion of females $\left(76 v .62 \%, \chi^{2}=8.207\right.$, d.f. $=1, P=0.004)$. Finally, cases or controls missing any demographic data were removed to leave a clean data-set of 620 cases with 1240 matched controls. The primary diagnoses of the cohort are shown in Table 1. Demographic frequencies, averages and between-group comparisons statistics are displayed in Table 2.

On average, those in the DNSD-S group were younger than the controls (33.4 v. 44.6 years). Age-at-diagnosis distributions are shown in Figs 1 and 2. The DNSD-S group distribution shows two peak ages at which patients generally received their diagnosis: one in their early 20s and one in their early 40s. Exploring this further, we split the DNSD-S group by median (31 years) and reran chi-square comparisons. Ethnicity showed no group differences above or below age 31 years. For gender, those in the DNSD-S group were overall still more likely to be female, but the proportion of males who received a diagnosis of DNSD-S significantly increased after 31 years of age, from 19.4 to $28.3 \%\left(\chi^{2}=6.794\right.$, d.f. $=1, P=$ $0.009, \varphi=0.009$ ). We did not compare temporal variables because these will be age dependent.

Historic mental and neurological conditions both showed significant differences between our groups, which confirmed the decision to further break down these two categories from the CRIS and HES databases. All prior diagnoses that reached significance were input into a logistic analysis alongside demographic variables. The results, including odds ratios, are displayed in Table 3. Individuals with DNSD-S were identified by this model with a sensitivity of $84.4 \%$, and controls were correctly discriminated with a specificity of $92.6 \%$ (overall correct classification: $89.8 \%$ ).

\section{Discussion}

This is the first study investigating demographic phenotypic differences between individuals with DNSD-S and those with other psychiatric diagnoses. Those with DNSD-S were more likely to be female (76 v. 47\%), of White ethnicity (77 v. 60\%), married or 


\section{Table 1 Primary diagnoses for the cohort}

\begin{tabular}{|c|c|c|}
\hline & Frequency, $n$ & $\%^{\mathrm{a}}$ \\
\hline \multicolumn{3}{|l|}{ DNSD-S group } \\
\hline Dissociative seizures & 620 & 100 \\
\hline \multicolumn{3}{|l|}{ Control group } \\
\hline Psychosis & 381 & 30.7 \\
\hline Depressive/mood disorder & 185 & 14.9 \\
\hline $\begin{array}{l}\text { Mental and behavioural disorders due to } \\
\text { use of alcohol and drugs }\end{array}$ & 173 & 14.0 \\
\hline $\begin{array}{l}\text { Anxiety disorder (phobic/general/OCD/ } \\
\text { PTSD) }\end{array}$ & 107 & 8.6 \\
\hline Mania/bipolar disorder & 106 & 8.5 \\
\hline Dementia & 57 & 4.6 \\
\hline $\begin{array}{l}\text { Hyperkinetic/conduct/unspecified } \\
\text { childhood-onset disorder }\end{array}$ & 57 & 4.6 \\
\hline Personality disorders & 65 & 5.2 \\
\hline $\begin{array}{l}\text { Other organic mental disorder/condition } \\
\text { (e.g. delirium/unspecified) }\end{array}$ & 32 & 2.6 \\
\hline Developmental disorders & 17 & 1.4 \\
\hline Eating disorder & 13 & 1.0 \\
\hline Delirium & 7 & 0.6 \\
\hline Dissociative other/motor & 6 & 0.5 \\
\hline $\begin{array}{l}\text { Other somatoform disorders (neurasthenia } \\
\text { or depersonalisation) }\end{array}$ & 6 & 0.5 \\
\hline $\begin{array}{l}\text { Unspecified mental disorder with } \\
\text { physiological dysfunction }\end{array}$ & $<5^{b}$ & $<4^{\mathrm{b}}$ \\
\hline \multicolumn{3}{|c|}{$\begin{array}{l}\text { DNSD-S, dissociative neurological symptom disorder with non-epileptic seizures; OCD, } \\
\text { obsessive-compulsive disorder; PTSD, post-traumatic stress disorder. } \\
\text { a. Percentages (\%) are of the total cohort or control group. } \\
\text { b. To maintain confidentiality, frequency values considered 'small' }(n<5) \text { were not } \\
\text { shown individually. }\end{array}$} \\
\hline
\end{tabular}

cohabitating (34v. 14\%) and to live in areas of lower socioeconomic status (25.7 v. 29.5\%).

Our study identified two peak ages at diagnosis. The first peak, during the patients' early 20 s, has been previously documented by a number of studies. ${ }^{1}$ The second peak, in their late 40 s, is of particular interest. Differences between early- and late-onset DNSD-S have been explored in a cohort of 241 patients, which showed that individuals with late-onset DNSD-S were more likely to be male and have severe physical health problems. ${ }^{17}$ Our results confirm that, although women are overall four times more likely to have DNSD-S than men, significantly more men were diagnosed with DNSD-S over the median age of 31 years $(61 \%)$ compared with women (49\%). In this regard, it must be emphasised that our study reported age at diagnosis rather than age at symptom onset and for this reason the two peaks do not necessarily reflect a different underlying aetiology or syndromic differences. However, Goldstein and colleagues ${ }^{8}$ found a similar trend in terms of age at onset, supporting the intriguing possibility of genuine aetiological differences.

In terms of medical history and comorbidities, compared with psychiatric controls, people with DNSD-S were 12 times more likely to have a previous diagnosis of a paroxysmal neurological condition such as epilepsy, and 10 times more likely to have had a diagnosis of a dissociative disorder, including dissociative amnesia, motor and anaesthesia symptoms. This may reflect a previous misdiagnosis of epilepsy ${ }^{4}$ but also the close relationship between DNSD-S and other dissociative disorders.

The rate of concomitant psychiatric diagnoses is another important finding of our study. Historically, people with DNSD-S have been considered 'complex' because of the high rates of concomitant psychiatric problems. ${ }^{18}$ Our study found that one in two in the DNSD-S group had a concomitant psychiatric problem but this is not statistically different from our psychiatric control group, pointing out that individuals with DNSD-S do not present a more complex psychiatric comorbidity pattern compared with those with other psychiatric disorders. More specifically, previous research has shown anxiety disorders (such as post-traumatic stress disorder, PTSD), depression and personality disorder to be common in people with DNSD-S. ${ }^{10,13}$ Our results suggest that they are common but they are not specific to patients with DNSD-S compared with those with other psychiatric conditions, as a personality disorder predictor remained non-significant, and both anxiety disorder and depression showed significant predictors, but odds ratios were minimal $(<1)$. Previous findings reflect the nature of the comparison, which most of the time was with epilepsy.

Finally, our study contributes to the debate about the role of somatisation and dissociation in people with DNSD-S. Previous authors have pointed out this role. ${ }^{19}$ However, our study found a low prevalence rate for somatisation the DNSD-S group that is not statistically different from the rate in the psychiatric controls. Although it may be the case that somatisation symptoms are common, ${ }^{19}$ our results show, clinically, that they may be underdiagnosed and that dissociation symptoms, episodic paroxysmal disorders, and symptoms, signs and abnormal clinical and laboratory

Table 2 Demographic frequencies, averages and comparison statistics of the DNSD-S group and controls

\begin{tabular}{|c|c|c|c|c|c|}
\hline & \multicolumn{2}{|c|}{ DNSD-S group, $n=620$} & \multicolumn{2}{|c|}{ Controls, $n=1240$} & \multirow{2}{*}{$\begin{array}{l}\text { Test, significance }(P), \text { effect size } \\
(\phi, \text { Cramer's } V \text { or Cohen's } d)\end{array}$} \\
\hline & Frequency, $n$ & $\%$ & Frequency, $n$ & $\%$ & \\
\hline Male* & 149 & 24.0 & 657 & 53.0 & $\chi^{2}=141.09$, d.f. $=1, P<0.001, \phi=0.275$ \\
\hline Female* & 471 & 76.0 & 583 & 47.0 & \\
\hline White* & 479 & 77.3 & 746 & 60.2 & $\chi^{2}=139.88$, d.f. $=5, P<0.001, V=0.274$ \\
\hline Black* & 59 & 9.5 & 344 & 27.7 & \\
\hline Mixed & 13 & 2.1 & 39 & 3.1 & \\
\hline Other ethnic group & 26 & 4.2 & 43 & 3.5 & \\
\hline Asian* & 18 & 2.9 & 68 & 5.5 & $\chi^{2}=139.88$, d.f. $=5, P<0.001, V=0.274$ \\
\hline Chose not to disclose* & 25 & 4.0 & 0 & 0.0 & \\
\hline Single* & 348 & 56.1 & 836 & 67.4 & $\chi^{2}=128.87$, d.f. $=5, P<0.001, V=0.263$ \\
\hline Married/cohabitating* & 213 & 34.4 & 173 & 14.0 & \\
\hline Divorced* & 26 & 4.2 & 104 & 8.4 & \\
\hline Widowed* & 6 & 1.0 & 47 & 3.8 & \\
\hline Separated* & 12 & 1.9 & 66 & 5.3 & \\
\hline \multirow[t]{2}{*}{ Chose not to disclose* } & 15 & 2.4 & 14 & 1.1 & \\
\hline & Mean & s.d. & Mean & s.d. & \\
\hline Age at diagnosis, years* & 33.4 & 13.8 & 44.6 & 17.5 & $\begin{array}{c}t=13.93, \text { d.f. }=1858, P<0.001, d=0.71 \text { (m.d. }=11.19 \\
95 \% \text { Cl 9.62-12.77) }\end{array}$ \\
\hline Socioeconomic status* (IMD score) & 25.7 & 14.1 & 29.5 & 11.1 & $\begin{array}{c}t=6.34, \text { d.f. }=1858, P<0.001, d=0.29 \text { (m.d. }=3.79 \\
95 \% \text { Cl } 2.62-4.96)\end{array}$ \\
\hline
\end{tabular}




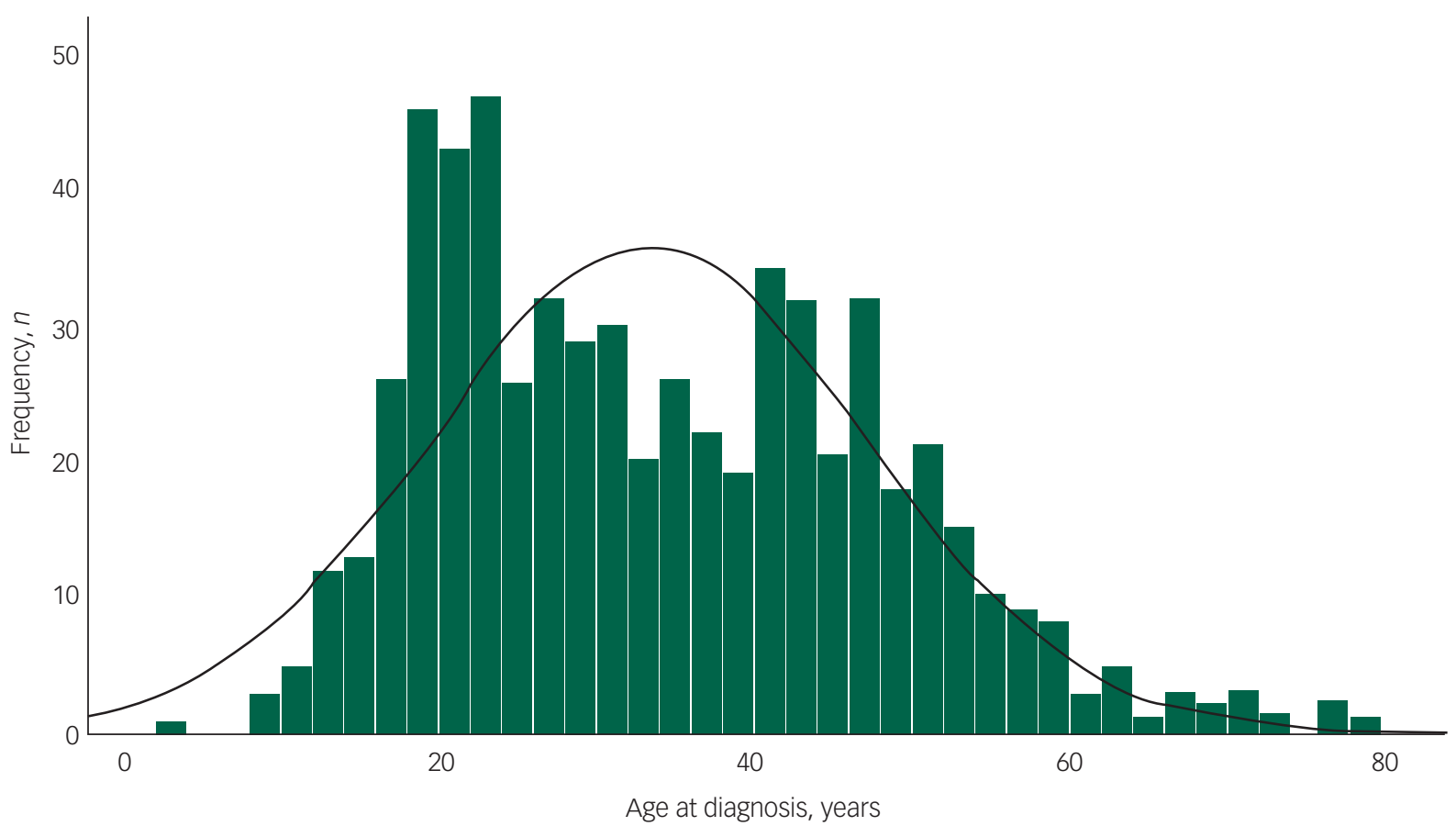

Fig. 1 Age at diagnosis of dissociative neurological symptom disorder with non-epileptic seizures (DNSD-S) in the cases group.

findings 'not elsewhere classified' may be just as, if not, more important. However, we should add the caveat that our sample potentially missed patients who had received an ICD diagnosis code of 'mixed dissociative disorder' that included dissociative seizures and who could have potentially showed greater somatisation. Regardless, this is an interesting finding, given the high associations between dissociative mechanisms and adverse events and trauma both prevalent in the DNSD-S population. ${ }^{20}$

\section{Limitations}

We should bear in mind that our findings will be somewhat influenced by the data's source; for example, hospital primary care data will not capture lesser medical conditions that do not require hospital attendance. Ideally, we would have analysed the incidence of medical conditions rather than dichotomously recording whether a condition had occurred in the past. However, we did not want to run the risk of being too reductionist when dealing with complex

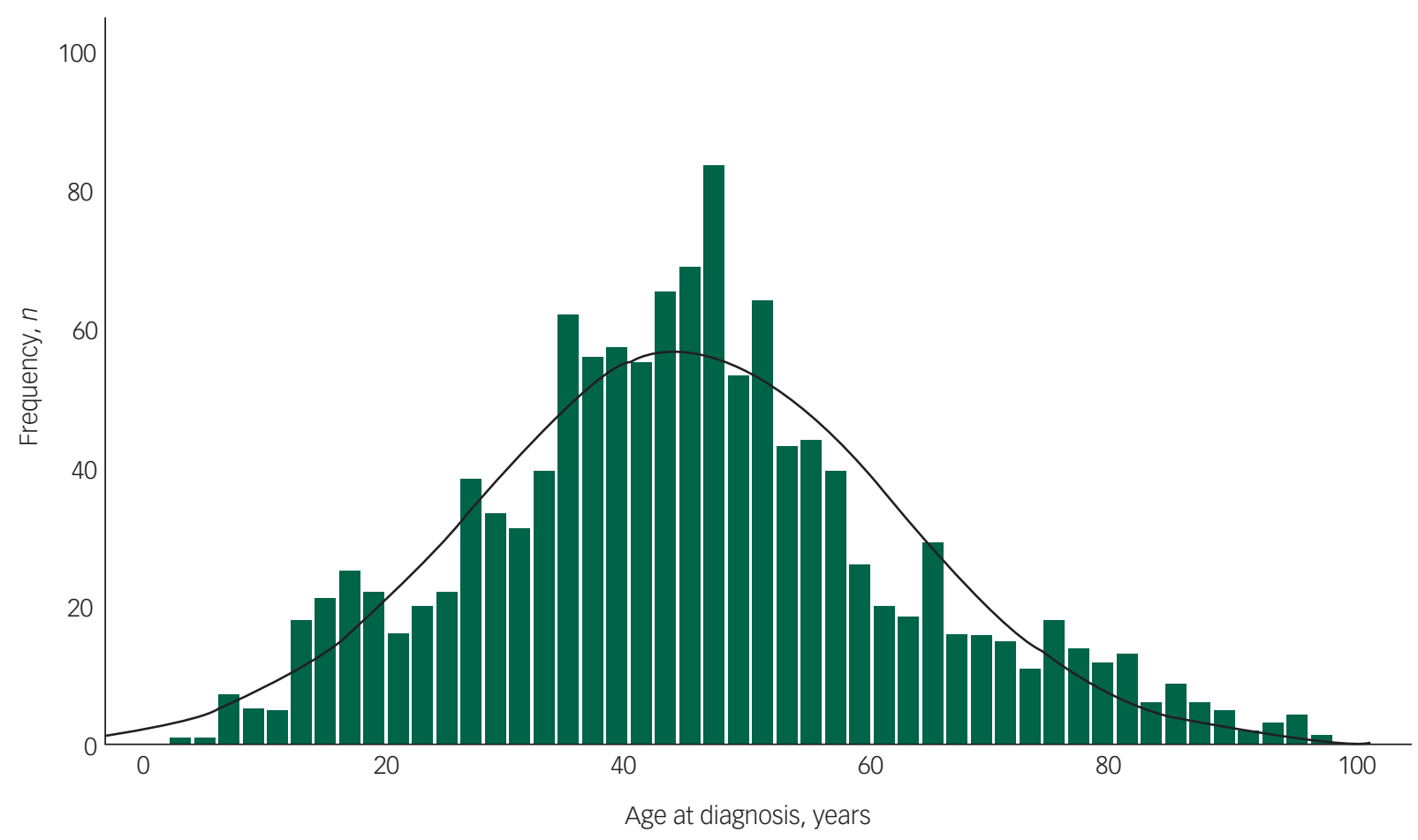

Fig. 2 Age at diagnosis of psychiatric disorders in the control group. 


\begin{tabular}{|c|c|c|c|c|c|c|c|c|}
\hline & \multirow[b]{2}{*}{ B } & \multirow[b]{2}{*}{ s.e. } & \multirow[b]{2}{*}{ Wald } & \multirow[b]{2}{*}{ d.f. } & \multirow[b]{2}{*}{$P$} & \multirow[b]{2}{*}{$O R^{b, c, d}$} & \multicolumn{2}{|c|}{$95 \% \mathrm{Cl}$ for $\operatorname{Exp}(\mathrm{B})$} \\
\hline & & & & & & & Lower & Upper \\
\hline Age at diagnosis, years* & -0.056 & 0.007 & 61.918 & 1 & 0.000 & 0.945 & 0.932 & 0.959 \\
\hline IMD score* & -0.015 & 0.007 & 5.368 & 1 & 0.021 & 0.985 & 0.972 & 0.998 \\
\hline Female* & 1.082 & 0.188 & 32.957 & 1 & 0.000 & 2.951 & 2.039 & 4.269 \\
\hline Ethnicity & & & 7.116 & 5 & 0.212 & & & \\
\hline White & -0.343 & 0.247 & 1.938 & 1 & 0.164 & 0.709 & 0.437 & 1.150 \\
\hline Black & -0.189 & 0.463 & 0.167 & 1 & 0.683 & 0.828 & 0.334 & 2.051 \\
\hline Mixed & 0.174 & 0.414 & 0.176 & 1 & 0.675 & 1.190 & 0.528 & 2.680 \\
\hline Other* & -1.013 & 0.439 & 5.331 & 1 & 0.021 & 0.363 & 0.154 & 0.858 \\
\hline Asian & 20.3997 & 7060.346 & 0.000 & 1 & 0.998722 & 2942556.108 & 0.000 & \\
\hline Marital status* & & & 42.050 & 5 & 0.000 & & & \\
\hline Single* & 1.374 & 0.231 & 35.413 & 1 & 0.000 & 3.953 & 2.514 & 6.215 \\
\hline Married/cohabitating & -0.060 & 0.382 & 0.025 & 1 & 0.874 & 0.941 & 0.445 & 1.991 \\
\hline Divorced & -0.121 & 0.544 & 0.049 & 1 & 0.824 & 0.886 & 0.305 & 2.574 \\
\hline Separated & 0.013 & 0.764 & 0.000 & 1 & 0.987 & 1.013 & 0.227 & 4.529 \\
\hline Widowed & -0.352 & 0.586 & 0.360 & 1 & 0.548 & 0.703 & 0.223 & 2.218 \\
\hline Diseases of the eye and adnexa & 0.637 & 0.338 & 3.553 & 1 & 0.059 & 1.892 & 0.975 & 3.670 \\
\hline Diseases of the ear and mastoid process & 0.893 & 0.579 & 2.382 & 1 & 0.123 & 2.443 & 0.786 & 7.592 \\
\hline Respiratory disease & -0.068 & 0.229 & 0.087 & 1 & 0.768 & 0.934 & 0.596 & 1.465 \\
\hline Diseases of the digestive system & -0.068 & 0.214 & 0.101 & 1 & 0.751 & 0.934 & 0.614 & 1.421 \\
\hline Diseases of the musculoskeletal system and connective tissue & 0.087 & 0.240 & 0.132 & 1 & 0.716 & 1.091 & 0.682 & 1.745 \\
\hline Diseases of the genitourinary system & 0.080 & 0.228 & 0.122 & 1 & 0.727 & 1.083 & 0.692 & 1.694 \\
\hline Symptoms, signs and abnormal clinical and laboratory findings, not elsewhere classified* & 1.410 & 0.230 & 37.500 & 1 & 0.000 & 4.095 & 2.608 & 6.430 \\
\hline Factors influencing health status and contact with health services & -0.294 & 0.216 & 1.843 & 1 & 0.175 & 0.745 & 0.488 & 1.139 \\
\hline Other degenerative diseases of the nervous system & -0.948 & 1.012 & 0.878 & 1 & 0.349 & 0.388 & 0.053 & 2.814 \\
\hline Episodic and paroxysmal disorders* & 2.533 & 0.243 & 108.916 & 1 & 0.000 & 12.590 & 7.824 & 20.260 \\
\hline Nerve, nerve root and plexus disorders & 0.743 & 0.506 & 2.157 & 1 & 0.142 & 2.102 & 0.780 & 5.668 \\
\hline Diseases of myoneural junction and muscle & 0.118 & 1.235 & 0.009 & 1 & 0.924 & 1.125 & 0.100 & 12.665 \\
\hline Cerebral palsy and other paralytic syndromes & -0.121 & 0.591 & 0.042 & 1 & 0.838 & 0.886 & 0.278 & 2.824 \\
\hline Other disorders of the nervous system & 0.157 & 0.438 & 0.129 & 1 & 0.719 & 1.170 & 0.496 & 2.760 \\
\hline Dementia & -20.1694 & 4861.184 & 0.000 & 1 & 0.997 & 0.000 & 0.000 & \\
\hline Mental and behavioural disorders due to use of alcohol and drugs* & -3.080 & 0.467 & 43.530 & 1 & 0.000 & 0.046 & 0.018 & 0.115 \\
\hline Psychosis* & -3.477 & 0.479 & 52.683 & 1 & 0.000 & 0.031 & 0.012 & 0.079 \\
\hline Mania/bipolar disorder & -20.6332 & 2745.750 & 0.000 & 1 & 0.994 & 0.000 & 0.000 & \\
\hline Depressive/mood disorder* & -2.323 & 0.302 & 59.332 & 1 & 0.000 & 0.098 & 0.054 & 0.177 \\
\hline Anxiety disorder (phobic/general/OCD/PTSD) * & -1.499 & 0.317 & 22.418 & 1 & 0.000 & 0.223 & 0.120 & 0.415 \\
\hline Dissociative other/motor* & 2.342 & 0.942 & 6.182 & 1 & 0.013 & 10.407 & 1.642 & 65.952 \\
\hline Personality disorders & -0.337 & 0.490 & 0.473 & 1 & 0.492 & 0.714 & 0.273 & 1.865 \\
\hline Mental retardation* & -2.101 & 0.924 & 5.174 & 1 & 0.023 & 0.122 & 0.020 & 0.748 \\
\hline Developmental disorders & -0.834 & 0.564 & 2.188 & 1 & 0.139 & 0.434 & 0.144 & 1.311 \\
\hline Hyperkinetic/conduct/unspecified child-onset disorder* & -1.503 & 0.516 & 8.491 & 1 & 0.004 & 0.223 & 0.081 & 0.611 \\
\hline Unspecified* & -0.572 & 0.275 & 4.335 & 1 & 0.037 & 0.565 & 0.330 & 0.967 \\
\hline Constant & 0.864 & 0.329 & 6.904 & 1 & 0.009 & 2.373 & & \\
\hline $\begin{array}{l}\text { DNSD-S, dissociative neurological symptom disorder with non-epileptic seizures; IMD, index of multiple de } \\
\text { disorder. } \\
\text { a. Diagnostic categories for mental and neurological conditions are from ICD-10. } \\
\text { b. Odds ratios indicate the predictiveness of developing DNSD-S given the presence of a variable charact } \\
\text { c. } \chi^{2}=1460.728, \text { d.f. }=39, P<0.001-\text { model coefficients. } \\
\text { d. } \chi^{2}=9.030, \text { d.f. }=8, P=0.340-\text { Hosmer-Lemeshow goodness-of-fit test. } \\
\text { * Indicates a significant contribution to the model. }\end{array}$ & $\begin{array}{l}\text { leprivation; } \\
\text { eristic. }\end{array}$ & & & & & & & \\
\hline
\end{tabular}

groups. Plus, this approach would have been more susceptible to reporter bias. Ideally, structured interviews would have been administered to glean these data, minimising reporter bias and allowing accurate diagnosis-frequency calculations. This is especially important in this area, given that there are generally issues with the reporting of complex disorders such as somatisation in routine clinical practice in general. However, owing to the retrospective nature and large numbers, this was not possible for the current study. The data were cross-sectional, and we therefore had to interpret temporal variables such as marital status with caution as they might change. We were unable to record the age at onset of a condition, only the age at which a patient was diagnosed. Next, although in our sample electroencephalogram (EEG) was the gold standard for diagnosis of DNSD-S, we did not check individual EEG results. Some individuals may have diagnoses based on experts' clinical judgement, which, naturally, increases risk of misdiagnoses. We should also bear in mind that patients with DNSD-S classified as a secondary diagnosis were removed from our sample. As a consequence, our results may not be valid in this population of patients. However, the numbers was small, and the purpose of this study was to focus on patients with DNSD-S as a primary diagnosis. Finally, we should reiterate that we used the ICD codes to group individuals with DNSD-S, which means that we will have missed patients with DNSD-S who also had other dissociative symptoms and were given the diagnosis 'F44.7 Mixed dissociative [conversion] disorders'.

\section{Implications}

Our findings aid other studies in clarifying the clinical and demographic characteristics of the DNSD-S patient population relative to the population with other psychiatric conditions. Clinicians should be aware of the increased incidence of DNSD-S diagnoses around the age of 40 , rather than simply in the early 20 s. The presence of prior paroxysmal symptoms, both neurological and dissociative, are more valid characteristics of DNSD-S than mood 
and anxiety conditions. Other pathological conditions may be important when identifying DNSD-S in neurological clinics, but this may not be so important when it comes to pathological aetiology and psychological treatment. Perhaps we place too great an emphasis and assume high psychiatric comorbidity in patients with DNSD-S because of past comparison with patients with epilepsy. The high predictivity of other neurological, functional and dissociative symptoms could suggest a common behavioural aetiology in these patients. It would be helpful for future studies to compare the behaviours and cognitions among DNSD-S patients to establish phenotypes. Any differences would be important to inform therapists of potential areas to target during treatment.

Abigail Smakowski $\mathbb{D}$, MSc, Neuropsychiatry Services, South London and Maudsley Hospital NHS Trust, UK; and Psychological Medicine, Institute of Psychiatry, Psychology and Neuroscience, King's College London, UK; Jacob S. Bird, MBBS, MRCPsych,

Department of Neuropsychiatry, St George's Hospital, South West London and St

George's Mental Health NHS Trust, London, UK; Megan Pritchard, MSC, National Institute for Health Research Maudsley Biomedical Research Centre, South London and Maudsley Hospital NHS Trust, King's College London, UK; Marco Mula (D), MD, PhD, FRCP, FEAN, Institute of Medical and Biomedical Education, St George's University of London, UK; and Atkinson Morley Regional Neuroscience Centre, St George's University Hospitals NHS Foundation Trust, London, UK

Correspondence: Abigail Smakowski. Email: abigail.1.smakowski@kcl.ac.uk First received 2 Sep 2020, final revision 11 Jan 2021, accepted 30 Jan 2021

\section{Data availability}

The data that support the findings of this study are available on request from the corresponding author. The data are not publicly available owing to patient confidentiality restrictions.

\section{Acknowledgements}

We thank our colleagues in the National Institute for Health Research (NIHR) Maudsley Biomedical Research Centre (BRC) Nucleus for their help with data extraction.

\section{Author contributions}

A.S. and J.S.B. conceived and designed the analysis; A.S., J.S.B. and M.P. collected the data and contributed data and analysis tools; A.S. performed the analysis; A.S., J.S.B. and M.M. wrote the paper.

\section{Funding}

This research received no specific grant from any funding agency, commercial or not-for-profit sectors.

\section{Declaration of interest}

None.

\section{References}

1 Asadi-Pooya AA, Sperling MR. Epidemiology of psychogenic nonepileptic seizures. Epilepsy Behav 2015; 46: 60-5.
2 Carson A, Lehn A. Epidemiology. In Handbook of Clinical Neurology (3rd Series): Vol. 139: Functional Neurologic Disorders (eds M. Hallett, J. Stone, A. Carson): 47-60. Elsevier, 2016.

3 Angus-Leppan $\mathrm{H}$. Diagnosing epilepsy in neurology clinics: a prospective study. Seizure 2008; 17: 431-6

4 Avbersek A, Sisodiya S. Does the primary literature provide support for clinical signs used to distinguish psychogenic nonepileptic seizures from epileptic seizures? J Neurol Neurosurg Psychiatry 2010; 81: 719-25.

5 Reuber M, Rawlings GH. Nonepileptic seizures - subjective phenomena. Funct Neurol Disord 2016; 139: 283-96.

6 Goldstein LH, Chalder T, Chigwedere C, Khondoker M, Moriarty J, Toone B, et al. Cognitive-behavioral therapy for psychogenic nonepileptic seizures: a pilot RCT. Neurology 2010; 74: 1986-94.

7 Reuber M. Psychogenic nonepileptic seizures: answers and questions. Epilepsy Behav 2008; 12: 622-35.

8 Goldstein LH, Robinson EJ, Reuber M, Chalder T, Callaghan H, Eastwood C, et al. Characteristics of 698 patients with dissociative seizures: a UK multicenter study. Epilepsia 2019; 60: 2182-93.

9 Benbadis SR, Agrawal V, Tatum WO 4th. How many patients with psychogenic nonepileptic seizures also have epilepsy? Neurology 2001; 57: 915-7.

10 Reuber M, Howlett S, Khan A, Grünewald RA. Non-epileptic seizures and other functional neurological symptoms: predisposing, precipitating, and perpetuating factors. Psychosomatics 2007; 48: 230-8.

11 LaFrance WC, Baker GA, Duncan R, Goldstein LH, Reuber M. Minimum requirements for the diagnosis of psychogenic nonepileptic seizures: a staged approach A report from the International League Against Epilepsy Nonepileptic Seizures Task Force. Epilepsia 2013; 54: 2005-18.

12 Dixit R, Popescu A, Bagic A, Ghearing GR, Hendrickson R. Medical comorbidities in patients with psychogenic nonepileptic spells (PNES) referred for video-EEG monitoring. Epilepsy Behav 2013; 28: 137-40.

13 Reuber M, Baker GA, Gill R, Smith D, Chadwick D. Failure to recognize psychogenic nonepileptic seizures may cause death. Neurology 2004; 62: 834-5.

14 Stewart R, Soremekun M, Perera G, Broadbent M, Callard F, Denis M, et al. The South London and Maudsley NHS Foundation Trust Biomedical Research Centre (SLAM BRC) case register: development and descriptive data. BMC Psychiatry 2009; 9: 51.

15 Perera G, Broadbent M, Callard F, Chang C-K, Downs J, Dutta R, et al. Cohort profile of the South London and Maudsley NHS Foundation Trust Biomedical Research Centre (SLaM BRC) Case Register: current status and recent enhancement of an Electronic Mental Health Record-derived data resource. BMJ Open 2016; 6(3): e008721.

16 Department for Communities and Local Government. English Indices of Deprivation 2015. Gov.uk, 2015 (https://www.gov.uk/government/statistics/ english-indices-of-deprivation-2015).

17 Duncan $R$, Oto $M$, Martin E, Pelosi A. Late onset psychogenic nonepileptic attacks. Neurology 2006; 66: 1644-7.

18 Carson AJ, Stone J, Warlow C, Sharpe M. Patients whom neurologists find difficult to help. J Neurol Neurosurg Psychiatry 2004; 75: 1776-8.

19 Reuber M, House AO, Pukrop R, Bauer J, Elger CE. Somatization, dissociation and general psychopathology in patients with psychogenic non-epileptic seizures. Epilepsy Res 2003; 57: 159-67.

20 Pick S, Mellers JD, Goldstein LH. Dissociation in patients with dissociative seizures: relationships with trauma and seizure symptoms. Psychol Med 2017; 47: $1215-29$ 